\title{
Development and management of ovarian hyperstimulation syndrome in spontaneous singleton pregnancy
}

\author{
Sercan Kantarcı ${ }^{1 *}$, Yaşam Kemal Akpak ${ }^{1}$, Betül Öztürk ${ }^{1}$, Serkan Oral $^{2}$, Atakan Emre ${ }^{1}$, \\ Alper İleri' ${ }^{1}$, Adnan Budak¹, Gökhan Tosun ${ }^{1}$, Hamdi İnan ${ }^{1}$, Mehmet Özeren'
}

\begin{abstract}
${ }^{1}$ Department of Obstetrics and Gynaecology, Tepecik Training and Research Hospital, Izmir, Turkey
${ }^{2}$ Department of Obstetrics and Gynaecology, Halic University, Istanbul, Turkey
\end{abstract}

Received: 28 July 2021

Revised: 01 September 2021

Accepted: 02 September 2021

*Correspondence:

Dr. Sercan Kantarc1,

E-mail: sercan.kntrc@gmail.com

Copyright: (c) the author(s), publisher and licensee Medip Academy. This is an open-access article distributed under the terms of the Creative Commons Attribution Non-Commercial License, which permits unrestricted non-commercial use, distribution, and reproduction in any medium, provided the original work is properly cited.

\begin{abstract}
Ovarian hyperstimulation syndrome (OHSS) is one of the most life-threatening complications of ovulation induction However, rarely, OHSS can develop spontaneously during pregnancy without induction of ovulation. It has been shown in the literature that some diseases such as polycystic ovary syndrome (PCOS), hypothyroidism, and pituitary adenoma may accompany spontaneous OHSS. Spontaneous OHSS should be included in the differential diagnosis in firsttrimester pregnancies with nausea, vomiting, and acute abdomen. The focus should be on preventing possible complications and initiating early treatment immediately after diagnosis.
\end{abstract}

Keywords: Spontaneous ovarian hyperstimulation syndrome, Ovarian torsion, Pregnancy first trimester complications, Ovarian cysts, Ovulation induction

\section{INTRODUCTION}

Ovarian hyperstimulation syndrome (OHSS) usually occurs in approximately $10 \%$ of cases due to ovulation induction. ${ }^{1}$ Although spontaneous OHSS is very rare, it has a later onset than iatrogenic OHSS. Although spontaneous OHSS is generally seen between 8-14 weeks, its etiopathogenesis has not been determined clearly. ${ }^{2}$

However, spontaneous OHSS can rarely occur due to the overstimulation of FSH receptors via glycoproteins such as FSH, LH, TSH, and hCG. ${ }^{3}$ Common risk factors are being younger than 35 years of age, low body mass index (BMI), a history of PCOS, and a previous history of OHSS. $^{4}$ Spontaneous OHSS may have complications secondary to hemoconcentration and electrolyte imbalance. $^{5}$ Iatrogenic OHSS is classified as mild, moderate, severe, and critical according to ultrasound and laboratory tests. Severe-critical OHSS may present severe clinical conditions such as acute renal failure, massive hydrothorax pericardial effusion, ARDS. While conservative treatments are preferred in managing patients who develop spontaneous OHSS, medical and surgical treatment options are also available depending on the severity and type of symptoms. ${ }^{6}$

\section{CASE REPORT}

An 18-year-old Turkish-origin primigravid spontaneous patient was referred to our center from an external center with complaints of persistent abdominal pain, bloating, dyspnea, nausea, and vomiting. The age of the first menstrual period is 14 and her periods are regular. The patient, who has no known comorbidity, history of drug use, and previous operation, is in the 10th week of spontaneous pregnancy without using an ovulation induction agent, according to the last menstrual period. The patient's height was $155 \mathrm{~cm}$, her weight was 59.3 
(body mass index: 24.6), and her abdominal circumference was $86.5 \mathrm{~cm}$. The ovaries were evaluated as normal in the pre-pregnancy ultrasound. The abdomen was distended in the patient's physical examination, bowel sounds, lung sounds, and heart sounds were evaluated as normal. In transabdominal ultrasound, the single intrauterine fetus was compatible with ten weeks according to Crown-rump length (CRL), fetal heart rate was positive (126 fhr), the bilateral multiloculated right ovary was 125 x $90 \times 127 \mathrm{~mm}$ in size, while left ovary was $125 \times 81 \times 103 \mathrm{~mm}$. The dimensions of both ovaries increased significantly, and the upper border of the ovary ended at the level of the umbilicus. While diffuse free fluid was observed in all quadrants, no signs of torsion were found on Doppler ultrasound. In the cardiac echo examination after the cardiology consultation, pericardial effusion was not observed, while the ejection fraction (EF) was $65 \%$ and reported as normal echo findings. Laboratory results are Hb:13.5 Htc:40.3 Beta hCG:201477, TSH:1.54, sT3:2.53, sT4:0.95, WBC:11.800, PLT:404,000, Na:130 mmol/l, Ca: $8.47 \mathrm{mg} / \mathrm{dl}, \mathrm{K}: 3.9 \mathrm{mmol} / \mathrm{l}, \mathrm{Cr}: 0.7 \mathrm{mg} / \mathrm{dl}$, Albumin:2.98 $\mathrm{g} / \mathrm{dl}$, and liver enzymes were found to be moderately high. As the tumor markers were CA125:129.7 U/ml, the patient was hospitalized, and a gynecological oncology consultation was requested. Since gynecological malignancy was not considered in the consultation, followup continued in our clinic for further examination and treatment.

The patient was evaluated as moderate OHSS. By monitoring the patient's intake and output, daily $1500 \mathrm{cc}$ NACL infusion plus Voluven $500 \mathrm{cc}$ infusion so 2 liters IV infusion, ceftriaxone $1 * 2 \mathrm{gr}$ IV, prophylactic $0.4 \mathrm{cc}$ SC low-molecular-weight heparin (LMWH) and, if needed,500 mg paracetamol IV infusion treatment were arranged. Recent intake and output, waist and abdominal circumference, weight, and fluid electrolyte levels were monitored. After the prophylactic LMWH treatment in the patient with normal coagulation test results, nosebleeds and no hemoconcentration were observed, so LMWH treatment was stopped. After ten days, the patient's clinical and laboratory values and intake-output balance tended to return to normal, and she was discharged to be called for control one week later. The patient came to her first control one week later. The ovary dimensions of the patient who did not have any active complaints were seen to have decreased by $70 * 85 \mathrm{~mm}$ and the left ovary by $79 * 90 \mathrm{~mm}$. Abdominal distension was decreased, free fluid was at the physiological level, and laboratory values were consistent with the week of gestation.

In the subsequent follow-ups, the double screening test was found to be low risk. The 2nd-trimester anomaly scan was normal. The patient's routine pregnancy follow-up, who also came regularly for subsequent pregnancy followups, was normal, and she delivered a $3200 \mathrm{~g}$ live male baby with 9 APGAR by normal spontaneous vaginal delivery at 39 weeks. All findings and imaging were within normal limits in the ultrasonography and laboratory evaluations performed at the 6th week of postpartum.

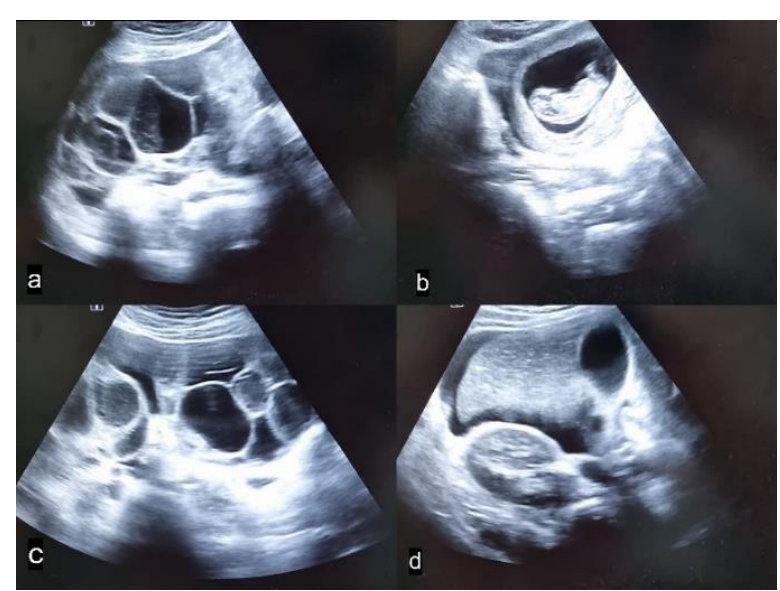

Figure 1: (a) Multiloculated 125x90x127 mm right ovary, (b) diffuse free fluid around the uterus (CRL 10W), (c) bilateral multiloculated big kissing ovary, (d) diffuse fluid in Morison's pouch.

\section{DISCUSSION}

Although spontaneous OHSS is usually seen between 8 and 14 weeks, in our case, it was diagnosed at an external center at the 10th week and referred to us. Although spontaneous OHSS is very rare, its pathophysiology has not yet been fully elucidated, but VEGF and VEGFR in the granulosa cells of the corpus luteum play a critical role in the development of OHSS. In addition, hCG and gonadotropins increase the amount of VEGF. ${ }^{7}$ Vasoactive substances secreted by the ovaries increase mesothelial hyperpermeability and cause fluid leakage from the intravascular space to the third space. hCG, estradiol, prolactin, histamine, and prostaglandins are mediators that aggravate OHSS. However, vasoactive substances secreted by the ovary, such as TNF-a, endothelin-1, and VEGF, which are better known today, increase vascular permeability. ${ }^{8,9}$ According to the De Leener classification, spontaneous OHSS consists of 3 types. Type 1 can cause recurrent OHSS associated with FSH receptor polymorphism. Type 2 is the most common type, hydatidiform mole accompanied by high hCG, and the type seen in multiple pregnancies. Type 3 is associated with hypothyroidism. ${ }^{10}$ Cases are primarily seen in patients with multiple pregnancies accompanied by high hCG levels, hydatidiform moles, or hypothyroidism characterized by high TSH levels. According to the literature, primary hypothyroidism characterized by high TSH levels or a very rare TSH-secreting adenoma may be associated with spontaneous OHSS. ${ }^{2}$ In our case, the beta hCG: 201477 value of the patient was higher than the week of gestation, but the thyroid hormones TSH:1.54 sT3:2.53 sT4:0.95 were found to be normal. Although our case did not have a history of hydatidiform mole, an appearance compatible with a mole was not observed on ultrasound. In addition, the postpartum placenta was sent to pathology and reported as a placenta compatible with its normal week. 
Known risk factors for OHSS are young age, low BMI, polycystic ovary syndrome, high-dose gonadotropin use, rapidly rising estradiol level, and a history of the previous OHSS. There are cases of spontaneous OHSS described in patients with polycystic ovary syndrome. ${ }^{11}$ Our patient had regular menstrual periods before pregnancy, no history of hair growth, and both ovaries were found to be normal on ultrasound.

Malignancy should also be considered in ovarian cysts that grow rapidly in a short time. Germ cell tumors should be considered in bilateral ovarian masses in adolescent pregnant women. ${ }^{12}$ In addition, CA125:129.7 was found in our patient. In Doppler ultrasound findings, ovarian cysts were evaluated as benign cystic because of the absence of solid components and their benign appearance of blood supply. In addition, malignancy was ruled out by requesting a gynecological oncology consultation. In addition, pregnancy luteoma and hyperreactio luteinalis (HL) can be considered in the differential diagnosis of OHSS. Pregnancy luteoma is a non-neoplastic solid ovarian mass lesion caused by excessive androgen secretion by the ovary, generally asymptomatic, seen in the last trimester and regressed with delivery. HL is a rare condition with bilateral multiloculated ovarian cysts that typically appear in the last trimester of pregnancy and regress with delivery. ${ }^{13}$

Ovarian torsion is a true gynecological emergency. Although it is seen in approximately $3 \%$ of all gynecological emergencies, $12-25 \%$ of these cases are pregnant. ${ }^{14}$ It usually presents with acute unilateral pain, accompanied by nausea, vomiting, defense, and rebound. Laparoscopy is one of the preferred approaches in adnexal torsion. Laparoscopy can be safely performed during pregnancy, especially in the early second trimester. In our case, we evaluated the ovarian blood supply with daily Doppler USG and excluded ovarian torsion and ovarian cyst rupture with the daily physical examination.

In the clinical presentation of OHSS, ascites, pleural effusion, and edema are observed due to fluid loss to the third space. It is this fluid loss that mainly causes mortality and morbidity. Hypovolemia due to fluid and protein loss, and the most severe complications related to hemoconcentration are hypercoagulability and decreased renal perfusion. ${ }^{15}$ Vital organs and systems can be severely damaged in critical OHSS. Acute renal failure, cardiac arrhythmia, respiratory failure, disseminated intravascular coagulation (venous and arterial thrombosis), and anuria can result in death. The pleural effusion transforms into massive hydrothorax with pericardial effusion. Sepsis and acute respiratory distress syndrome (ARDS) can further complicate the clinical picture. For this reason, as in our case, close monitoring of vital and fluid balance, albumin level, use of LMWH are critically important for the followup and prevention of OHSS complications.

Spontaneous OHSS is a very rare condition. A delay in diagnosis can lead to life-threatening complications.
Although there is no specific treatment, most cases require hospitalization. Hemodynamic close monitoring, intravenous albumin, crystalloid infusion, and thromboembolism prophylaxis constitute the basic treatment principles. ${ }^{11}$ OHSS should be considered in the differential diagnosis of patients with first-trimester nausea, vomiting, edema, abdominal distension, increased ovarian size, who did not receive ovulation induction, and should be followed closely for possible complications.

\section{CONCLUSION}

In our case, increased ovarian size, abdominal pain, bloating, nausea, vomiting and ascites were detected on ultrasound. Although she did not receive ovulation induction, she was thought to be clinically compatible with OHSS. She was followed closely for complications of ovarian torsion, electrolyte imbalance, pulmonary edema, renal failure, deep vein thrombosis. Fluid therapy was arranged according to the fluid intake and output, and anticoagulant therapy was started. It should be kept in mind that OHSS can also develop spontaneously. The importance of early diagnosis and treatment should be emphasized in terms of preventing life-threatening complications.

\section{Funding: No funding sources \\ Conflict of interest: None declared \\ Ethical approval: Not required}

\section{REFERENCES}

1. Ezra Y. Modern trends. Fertil Steril. 1994;61(3):411-22.

2. Ahmed Kamel RM. Spontaneous ovarian hyperstimulation syndrome in a naturally conceived singleton pregnancy. Fertil Steril. 2010;94(1):351.e1-4.

3. Mittal K, Koticha R, Dey AK Anandpara K, Agrawal $\mathrm{R}$, Sarvothaman MP, et al. Radiological illustration of spontaneous ovarian hyperstimulation syndrome. Polish J Radiol. 2015;80(1):217-27.

4. Di Carlo C, Savoia F, Fabozzi A, Gargano V, Nappi C. Case Report: A case of ovarian torsion in a patient carrier of a FSH receptor gene mutation previously affected by spontaneous ovarian hyperstimulation syndrome. Gynecol Endocrinol. 2014;3590:1-4.

5. Borna S, Nasery A. Spontaneous ovarian hyperstimulation in a pregnant woman with hypothyroidism. Fertil Steril. 2007;88(3):705.e1-3.

6. Di Carlo C, Savoia F, Gargano V, Sparice S, Bifulco G, Nappi C. Successful pregnancy complicated by spontaneous, familial, recurrent ovarian hyperstimulation syndrome: Report of two cases. Gynecol Endocrinol. 2013;29(10):897-900.

7. Oral S, Akpak YK, Karaca N, Savan K. The Importance of Prolactin Levels in Patients Treated with Cabergoline for the Prevention of OHSS: Is Cabergoline Really Effective in Patients with High 
Risk of OHSS? Open J Obstet Gynecol. 2015;05(06):344-9.

8. Binder H, Dittrich R, Einhaus F, Krieg J, Müller A, Strauss R, et al. Update on ovarian hyperstimulation syndrome: Part 1--Incidence and pathogenesis. Int $\mathrm{J}$ Fertil Womens Med. 2007;52(1):11-26.

9. Cabar FR. Ovarian hyperstimulation syndrome in a spontaneous singleton pregnancy. Einstein (Sao Paulo). 2016;14(2):231-4.

10. De Leener A, Montanelli L, Van Durme J, Chae H, Smits G, Vassart G, et al. Presence and absence of follicle-stimulating hormone receptor mutations provide some insights into spontaneous ovarian hyperstimulation syndrome physiopathology. J Clin Endocrinol Metab. 2006;91(2):555-62.

11. Zalel Y, Orvieto R, Ben-Rafael Z, Homburg R, Fisher $\mathrm{O}$, Insler V. Recurrent spontaneous ovarian hyperstimulation syndrome associated with polycystic ovary syndrome. Gynecol Endocrinol. 1995;9(4):313-5.
12. Krishnakumar S, Kuris S, Kaveri R, Joshi A, Krishnakumar R. Spontaneous OHSS in a Young Adolescent: A Diagnostic Dilemma. J Obstet Gynecol India. 2020;70(3):237-9.

13. Haimov-Kochman R, Yanai N, Yagel S, Amsalem H, Lavy Y, Hurwitz A. Spontaneous ovarian hyperstimulation syndrome and hyperreactio luteinalis are entities in continuum. Ultrasound Obstet Gynecol. 2004;24(6):675-8.

14. Rackow BW, Patrizio P. Successful pregnancy complicated by early and late adnexal torsion after in vitro fertilization. Fertil Steril. 2007;87(3):697.e9-12.

15. Practice T, Medicine R. Ovarian hyperstimulation syndrome. Fertil Steril. 2008;90(5 Suppl):188-93.

Cite this article as: Kantarcı S, Akpak YK, Öztürk B, Oral S, Emre A, İleri A, et al. Development and management of ovarian hyperstimulation syndrome in spontaneous singleton pregnancy. Int $\mathbf{J}$ Reprod Contracept Obstet Gynecol 2021;10:3954-7. 